
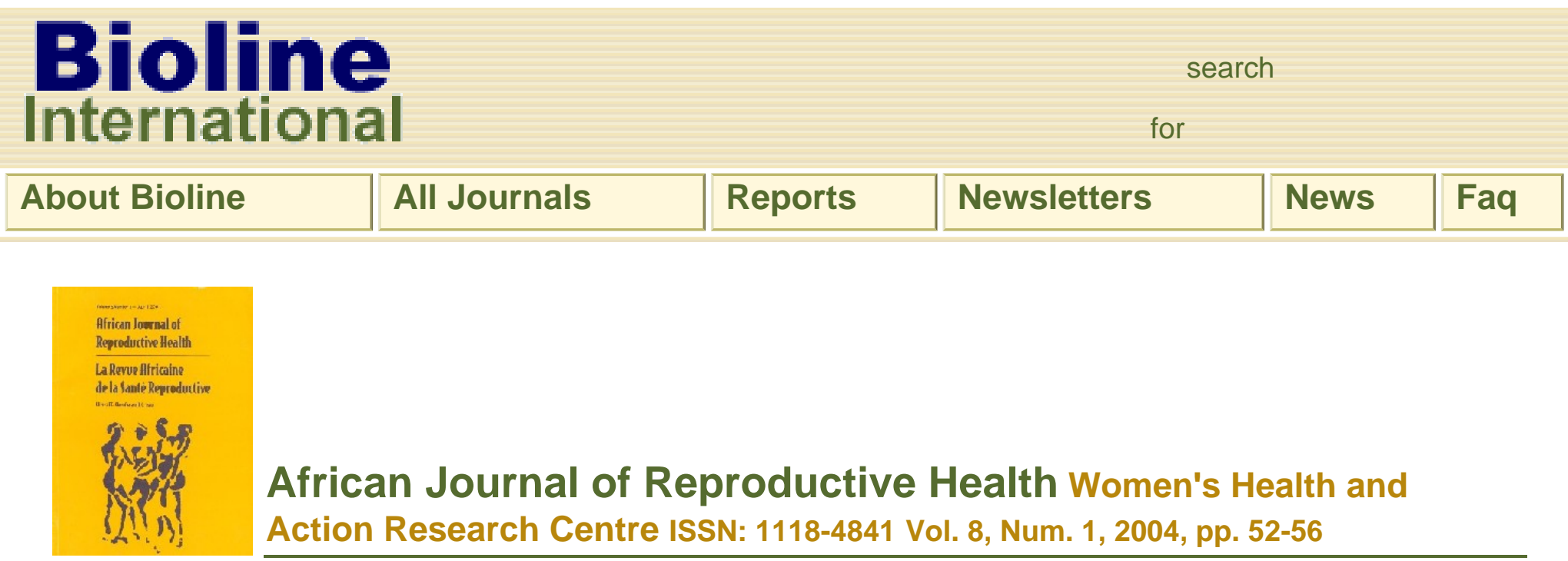

African Journal of Reproductive Health Women's Health and Action Research Centre ISSN: 1118-4841 Vol. 8, Num. 1, 2004, pp. 52-56

African Journal of Reproductive Health, Vol. 8, No. 1, April, 2004 pp. 52-56

\title{
Planning for Sustainable Access to Technology: An Essential Element of Safe Abortion Care
}

Nina Frankel ${ }^{1}$ and Nancy Nyaga ${ }^{2}$

1Senior Advisor for Reproductive Health Technology Introduction at Ipas, 300 Market Street, Suite 200, Chapel Hill, NC 27516 USA. Tel: 919.967.7052; E-mail: nfrankel@unc.edu ${ }^{2}$ Coordinator for the Ipas Africa Alliance for Women's Reproductive Health and Rights, Nairobi, Kenya.

Correspondence: Ms. Nina Frankel, Senior Advisor for Reproductive Health Technology Introduction at Ipas, 300 Market Street, Suite 200, Chapel Hill, NC 27516 USA. Tel: 919.967.7052; E-mail: frankeln@ipas.org

\section{Code Number: rh04009}

\section{One Clinic's Story}

Uterine evacuation services in the public clinic were going well. After a long and concerted effort, systems for providing manual vacuum aspiration (MVA) services to the abortion and postabortion care patients were in place. Doctors and midwives were trained, and an international donor had provided several MVA instruments. The clinic managers were pleased to be providing the improved services in accordance with new national protocols prescribing that sharp curettage services be replaced with MVA. The clinic staff worked with members of the local community to explain that MVA procedures are simple and safe and that women could now gain access to care more quickly, as they would no longer need to travel to the operating theatre of the regional hospital.

Within months, community health workers were referring more women to the clinic, and the 
number of MVA procedures performed had increased. The clinic was saving money, and fees for patients were lower. But then, the MVA kits began to age. After they were used for a large number of procedures, it was clear that replacement instruments would be needed. The clinic manager requested replacements but was informed by the procurement officer that MVA was not included on the "essential equipment" list and, thus, additional authorisation would be necessary. The clinic manager then learned that the current district health budget also did not provide for purchase of new instruments and any expenditure would need to wait until the following year. Because a sustainable system for obtaining MVA instruments was not in place, the clinic was forced to suspend the service and lose the significant improvements that had been made in care for the clinic's patients.

Key Words: Manual vacuum aspiration, logistics, supply chain, abortion technology

\section{Introduction}

In the past decade, new policies and programmes have been introduced in Africa to provide post-abortion care and, in some countries, safe, legal abortion services. Unfortunately, the programmes have often neglected systems to ensure that the required technologies for uterine evacuation are available when and where they are needed. Reproductive health providers, managers and advocates often recognise the importance of these technologies, but may not have the expertise, mandate or resources to take the complex steps required to ensure their sustainable supply. They also may encounter political sensitivities and reluctance by national decision-makers, as well as some donors, to facilitate access to abortion technologies in the same way that they facilitate other health technologies. ${ }^{*}$

**Some donors, e.g., the US Agency for International Development, have provided significant support to post-abortion care programmes and even endorse manual vacuum aspiration (MVA) as a preferred technology, but have a policy of not funding purchase of the instruments.

Despite these obstacles, financial and human resources must be committed to ensuring sustainability, i.e., the long-term, continuous and routine access to technologies. Without technologies in place on a reliable basis, women are much less likely to receive good quality care, or may be denied care altogether. The absence of technologies effectively violates women's human rights as defined in international treaties and conventions, including their rights to health and to the benefits of scientific progress - both of which imply the right to information on their treatment options and to treatment with the safest and most effective modern technologies.

The purpose of this paper is to present a con- ceptual framework designed to facilitate planning for the actions required to ensure sustainable access to preferred modern uterine evacuation technologies. Obtaining government or donor funds to purchase technologies and seeking the lowest priced technologies on the market is sometimes seen as the primary way to increase access. However, many other steps are required for sustainability. It is very important that technologies meet quality standards, be in compliance with applicable regulatory requirements, and be supported by a reliable distribution system. 
Planning and coordinating the necessary actions for sustainability is a process that requires involvement of multiple actors, including programme managers, medical leaders, manufacturers, local commercial distributors of medical equipment and drugs, health system agencies responsible for procurement, high level officials in the health system responsible for planning and budgets, and, in many cases, donor agencies. In order to move the process forward, one or more of these actors must show leadership to engage the other partners.

\section{A Framework for Achieving Technology Sustainability}

Technology sustainability depends on fulfilling a number of pre-conditions, often simultaneously:

- Trained providers who are prepared not only to provide clinical care but also to promote services and the need for the preferred technologies.

- Medical guidelines and protocols setting standards for preferred technologies.

- Policies that facilitate importation and distribution of the technologies.

- Financial resources and mechanisms for procurement and distribution.

In the remainder of this paper, we will examine these pre-conditions individually. The framework presented here assumes that a generally supportive policy environment exists; with laws and policies in place that permit induced abortion in some circumstances as well as leaders in the public health sector who support abortion to the extent of the law or at least support postabortion care. The more restrictive the laws and policies, and the less support from high level policymakers, the more likely it is that efforts to achieve sustainable access to technologies will encounter obstacles in each of the areas described below. Creative approaches and persistence may be needed to build support and overcome these obstacles.

\section{Trained Providers}

Introduction of new technologies depends on achieving a critical mass of trained providers who are able to use them safely and effectively. Training in uterine evacuation procedures and the other elements of comprehensive, woman-centred abortion care can take place in a variety of ways, including both in-service, on-the-job training for current providers and pre-service training for those who are still students. Where regulations permit, not only obstetricians/gynaecologists and other physicians, but also nurses, midwives and other mid-level providers can be trained in these procedures. Unfortunately, agencies often offer excellent training but give little attention to how the trained providers will be able to obtain the required technologies continuously. A plan for this purpose, with involvement of appropriate partners, should be integral to any training programme.

Trained providers can also be important allies in promoting sustainable access to technology. 
When well-respected providers are trained, they may also facilitate the development of medical norms and guidelines and treatment protocols in their own institutions and nationally. As individuals, they can be effective champions for safe abortion care and, even if they do not directly affect policies of the health ministry and other government bodies, they may influence the introduction of new technologies and their acceptance by policymakers, other providers and the public. Leading providers may also play a role in assessing new technologies for regulatory institutions.

\section{Medical Guidelines and Protocols Setting Standards}

During the development of medical guidelines and standard treatment protocols, identifying the technologies that are most appropriate for providers at various levels of the health system is part of the decision process. These decisions need to take into account international guidance on preferred techno-logies, the availability of trained providers, and the capacity of the existing service delivery infra-structure.

Quality standards for abortion-related care have recently been defined in the new guidance from the World Health Organization (WHO), including the technologies that best meet these standards. The preferred technologies for early abortion are vacuum aspiration, performed with either electric vacuum aspiration (EVA) or manual (MVA) instruments, and pharmaceutical abortifacients such as mifepristone and misoprostol, often referred to as medical abortion. The WHO guidance considers vacuum aspiration to be the preferred surgical technique for abortion up to 12 completed weeks of pregnancy. It further states that where D \& C (dilatation and curettage) is currently practiced, all possible efforts should be made to replace it with vacuum aspiration to improve the safety and quality of care, and that where no abortion-related services are currently offered, vacuum aspiration should be introduced rather than $D$ \& $C$. Even in places where induced abortion is legally restricted, the availability of vacuum aspiration technology is strongly indicated for those early induced abortions that are legally permitted as well as for the treatment of incomplete abortion.

MVA technology is particularly well-suited for low- resource settings. It consists of a durable plastic hand-held aspirator and a set of tubes, or "cannulae" of various sizes. The aspirator produces a vacuum when its pinch valves are manually locked and its plunger is pulled into a locked position. To perform an MVA procedure, a cannula is inserted through the cervical os into the uterine cavity. The aspirator is then attached to the cannula. When the pinch valves are released, the vacuum aspirates the contents of the uterus into the aspirator. MVA is inexpensive, portable and re-usable, and it does not require electricity, an operating theatre or other expensive infrastructure. Where regulations permit, mid-level providers such as nurses, midwives and clinical officers can be trained to perform the procedure, reducing the need for doctors.

Medical methods of abortion - mainly mifepristone and misoprostol - are also becoming available in some countries and hold significant promise. Different regimens involving oral and/ or vaginal administration are used at different stages of pregnancy. According to the new WHO guidance, medical methods of abortion have been proved to be safe and effective, and have 
also proved acceptable in low-resource settings. Surgical back-up is needed, however, for cases of incomplete or failed abortions.

This paper will primarily discuss sustainability of MVA instrument supply, although the general principles are also relevant for EVA and medical abortion, contraceptives and other critical reproductive health technologies.

\section{Policies that Facilitate Importation and Distribution of the Technologies}

Availability of abortion technologies can be facilitated if they are not only identified in medical guidelines but are incorporated into a country's lists of essential services, equipment, and/or drugs, which guide procurements in the public sector. When a service or technology has been defined as "essential," it is easier to demonstrate the need for procuring it and including it in budget line items.

Technologies also must be registered with the national authorities that regulate pharmaceuticals and medical devices in order to be used in the public or private sector. Registration procedures vary from country to country, ranging from requirements that are minimal to those that are extremely complex. Many African countries use European Union (EU) and/or United States Food and Drug Administration (USFDA) regulations as the foundation for their own requirements. Registrations must also be renewed periodically; it is therefore important that registration and other regulatory requirements are monitored.

Importation taxes and customs regulations and fees can often raise the cost of technologies substantially. Health ministry leaders can sometimes negotiate special importation status and/or fee exemp-tions.

Local commercial distributors of medical equipment and drugs are usually very familiar with the requirements for registration and importation and knowledgeable about how decisions are made. They can often play a very helpful role in fulfilling these requirements, working with manufacturers of the technologies.

\section{Financial Resources and Mechanisms for Procurement and Distribution}

Procurement systems for the public sector vary in the degree to which they are centralised or decentralised. As a result of health sector reform, it is increasingly common for regional or local health institutions to have authority to procure supplies and equipment according to their own needs. Such decentralisation can facilitate access to technologies if the local authority is committed to the service and is well prepared to address the logistics of distribution. On the other hand, decentralisation can also make it more difficult to assure consistent access to technologies and services across the country. Even in decentralised systems, however, most federal governments use a number of mechanisms to retain some control. For instance, in some countries, the federal government negotiates the tenders and pricing structures, leaving individual facilities to make their own purchasing decisions. In other countries, the central government actually makes the purchases and then distributes the supplies as needed to public 
sector facilities. If this system does not operate efficiently, however, including the systems for requisitioning and distributing supplies, it is difficult to assure reliable access to the technologies.

Commercial distributors of medical instruments, drugs and other supplies play a key role in assuring sustainable access in both the public and private sectors. As previously noted, they can help in addressing registration and importation requirements. They are familiar with local markets and may have established distribution networks, with geographical coverage that extends beyond major cities to rural health centres and hospitals. They maintain relationships with hospitals, professional associations and other key stakeholders; and, because they achieve a financial return, have the ability to purchase and stock supplies so that they are available when needed. Distributors sell to private sector facilities and health providers, and they respond to public sector tenders. Some distributors even provide training in the use of the products that they sell.

Even with systems in place, the initial cash outlay for public sector health systems and NGOs that serve low-income women can be an obstacle to purchasing the required technologies. Historically, international donors that support reproductive health have donated equipment and supplies, or subsidised the costs. However, reliance on donor funds does not assure long-term sustainability. The case must be made for financing uterine evacuation technologies from national or local health budgets. Decision-makers should be informed that MVA instruments, designed to withstand repeated use and processing with no loss in performance, allow the initial cost of the instruments to be spread over time, resulting in a very low cost per procedure for a life-saving intervention. Medical abortion methods are still relatively costly, although prices vary considerably depending on the method and the manufacturer. While it is critical for services to remain affordable to the poorest women, fees for abortion-related services may help defray the costs of the technologies.

\section{Conclusion}

As is true for other health care commodities and equip-ment, reliable local availability of MVA instruments and other preferred abortion technologies through normal distribution channels is essential to ensure consistent and high quality services. Achieving this goal requires cooperation and coordination among multiple stakeholders. Trained and committed providers, supportive health system administrators and motivated commercial distributors have leading roles to play, with inputs from manufacturers, donor agencies, women's health advocates and others. Once a system is put in place, it needs to be regularly monitored, evaluated and improved, using key indicators to assess whether technologies are available to providers and patients in the most timely and efficient way.

The availability of affordable technologies for abortion and post-abortion care is critical to women's health. Technologies such as manual vacuum aspiration and, increasingly, medical abortion, can expand access to care at the local level. Because these technologies can be provided by mid-level providers in primary health care settings, they reduce the need for doctors and expensive hospital-based care. By developing and implementing plans for sustainable 
Bioline International Official Site (site up-dated regularly)

access to preferred uterine evacuation technologies, health systems will be able to offer options to women with unwanted pregnancies, improve their reproductive health, and in many cases save their lives.

\section{Acknowledgements}

The authors would like to thank Nadine Burton and Barbara Crane for their contributions to the ideas presented in this paper.

\section{References}

1. Setty-Venugopal V, Jacoby $R$ and Hart C. Family planning logistics: strengthening the supply chain. Population Reports 2002; Series J, No. 51. http://www.infoforhealth.org/pr/ j51edsum.shtml

2. Population Action International. Meeting the Challenge: Securing Reproductive Health Supplies. John Snow Inc., 2001. http://www.populationaction.org/resources/publications/ commodities/PDFs/PAl 09 Eng.pdf

3. UNFPA. Reproductive Health Commodity Security: Partnerships for Change. New York, 2001. http://www.unfpa.org/upload/lib pub file/135 filename rhcstrategy.pdf

4. Family Planning Logistics Management/John Snow Inc. Programs that Deliver: Logistics' Contributions to Better Health in Developing Countries. Arlington, Va.: Family Planning Logistics Management/John Snow, Inc, for USAID, 2000. http://deliver.jsi. com/2002/Pubs/Pubs Policy/Programs That Deliver/TOC/index.cfm

5. Bates James, Yasmin Chandani, Kathryn Crowley, John Durgavich and Sandhya Rao. Implications of Health Sector Reform for Contraceptive Logistics: A Preliminary Assessment for Sub-Saharan Africa. Arlington, Va.: Family Planning Logistics Management/John Snow Inc., for USAID, 2000. http://deliver.jsi.com/PDF/pa/ HSR SynthesisPaper2000.pdf

6. Family Planning Logistics Management/John Snow, Inc. The Logistics Handbook: A Practical Guide for Supply Chain Managers in Family Planning and Health Programs. Arlington, Va.: Family Planning Logistics Management/John Snow, Inc, for USAID, 2000. http://deliver.jsi.com/PDF/g\&h /LogisticsHandbook.pdf

7. Owens Richard C Jr and Timothy Warner. Concepts of Logistics System Design. Arlington, Va.: Family Planning Logistics Management/John Snow Inc., for USAID, 1996. http://deliver.jsi.com/pdf/G\&H/logistics system design2.pdf.

8. World Health Organization. Safe Abortion: Technical and Policy Guidance for Health Systems._www.who.int/ reproductive-health/publications/Safe-Abortion.pdf

(C) Women's Health and Action Research Centre 2004 
Bioline International Official Site (site up-dated regularly)

\begin{tabular}{|c|c|c|}
\hline Home & Resources & Mailing List \\
\hline
\end{tabular}

(C) Bioline International, 1989 - 2004, Site last up-dated on 20-Jan-2005. 